\title{
Small-Molecule Inhibitors Targeting Protein SUMOylation as Novel Anticancer Compounds
}

\author{
Yanfang Yang, Zijing Xia, Xixi Wang, Xinyu Zhao, Zenghua Sheng, Yang Ye, Gu He, \\ Liangxue Zhou, Hongxia Zhu, Ningzhi Xu, and Shufang Liang
}

State Key Laboratory of Biotherapy and Cancer Center, West China Hospital, Sichuan University, and Collaborative Innovation Center for Biotherapy, Chengdu (Y.Ya., Z.X., X.W., X.Z., Z.S., Y.Ye., G.H., L.Z., N.X., S.L.); Departments of Nephrology (Z.X.) and Neurosurgery (L.Z.), West China Hospital, Sichuan University, Chengdu; and Laboratory of Cell and Molecular Biology, and State Key Laboratory of Molecular Oncology, Cancer Institute and Cancer Hospital, Chinese Academy of Medical Sciences, Beijing (H.Z., N.X.), People's Republic of China

Received February 26, 2018; accepted May 16, 2018

\section{ABSTRACT}

SUMOylation, one of post-translational modifications, is covalently modified on lysine residues of a target protein through an enzymatic cascade reaction similar to protein ubiquitination. Along with identification of many SUMOylated proteins, protein SUMOylation has been proven to regulate multiple biologic activities including transcription, cell cycle, DNA repair, and innate immunity. The dysregulation of protein SUMOylation and deSUMOylation modification is linked with carcinogenesis and tumor progression. The SUMOylation-associated enzymes are usually elevated in various cancers, which function as cancer biomarkers to relate to poor outcomes for patients. Considering the significance of protein SUMOylation in regulating diverse biologic functions in cancer progression, numerous smallmolecule inhibitors targeting protein SUMOylation pathway are developed as potentially clinical anticancer therapeutics. Here, we systematically summarize the latest progresses of associations of small ubiquitin-like modifier (SUMO) enzymes with cancers and small-molecular inhibitors against human cancers by targeting SUMOylation enzymes. We also compared the pros and cons of several special anticancer inhibitors targeting SUMO pathway. As more efforts are invested in this field, smallmolecule inhibitors targeting the SUMOylation modification pathway are promising for development into novel anticancer drugs.

\section{Introduction}

The small ubiquitin-like modifier (SUMO) proteins, approximately with $11 \mathrm{kDa}$, covalently modify lysine (Lys) amino acid of a target substrate protein. Four major SUMO paralogues, including SUMO1, SUMO2, SUMO3, and SUMO4, are expressed in mammalian cells (Enserink, 2015). Most SUMOylation sites of a target protein reside in a consensus motif with the $\psi \mathrm{KxE}$ or $\psi \mathrm{KxE} / \mathrm{D}$ sequence ( $\psi$ is an aliphatic residue, $\mathrm{K}$ is the target Lys, $\mathrm{E} / \mathrm{D}$ is Asp or Glu, and $\mathrm{x}$ is any amino acid) (Yang et al., 2017). Except binding in consensus motif, SUMOs can noncovalently

This work was supported by the National 863 High Tech Foundation [Grant 2014AA020608], the National Key Basic Research Program of China [Grants 2013CB911303 and 2011CB910703], the National Natural Science Foundation of China [Grants 31470810 and 31071235], the Science and Technology Department of Sichuan Province [Grant 2017JY0232], and the Health and Family Planning Commission of Sichuan Province [Grant 17ZD045].

https://doi.org/10.1124/mol.118.112300. interact with proteins through targeting specific SUMOinteracting motifs (Hecker et al., 2006).

SUMOylation regulates multiple biologic processes including transcription, cell cycle, DNA repair, and innate immunity (Seeler and Dejean, 2003; Bettermann et al., 2012). A multistep enzymatic cascade reaction is involved in the biochemical process of protein SUMOylation (Fig. 1). First, four SUMO paralogues are synthesized as preproteins that are cleaved by sentrin-specific proteases (SENPs) to expose a diglycine (GG) motif on the carboxy-terminus. After that, an ATP-requiring activation, mediated by a heterodimeric SUMO-activating enzyme E1 (SUMO E1), generates a SUMO-SUMO E1 thioester. Then SUMO is transferred to the SUMO-conjugating enzyme E2 (SUMO E2) to form a thioester again. Next, a SUMO ligase E3 (SUMO E3) stabilizes the interaction between SUMO E2 and the substrate, and brings about an isopeptide bond between the SUMO C-terminus and a Lys within the target

ABBREVIATIONS: HCC, hepatocellular carcinoma; ML-792, [(1R,2S,4R)-4-[[5-[1-[(3-bromophenyl)methyl]pyrazole-3-carbonyl]pyrimidin-4-yl]amino]2-hydroxycyclopentyl]methyl sulfamate; PTM, protein post-translational modification; SAE1/2, SUMO-activating enzyme subunit 1/2; SENP(s), sentrinspecific proteases; SUMO, small ubiquitin-like modifier; SUMO E1, SUMO-activating enzyme E1; SUMO E2, SUMO-conjugating enzyme E2; SUMO E3, SUMO ligase E3; Ubc9, ubiquitin-conjugating enzyme 9. 


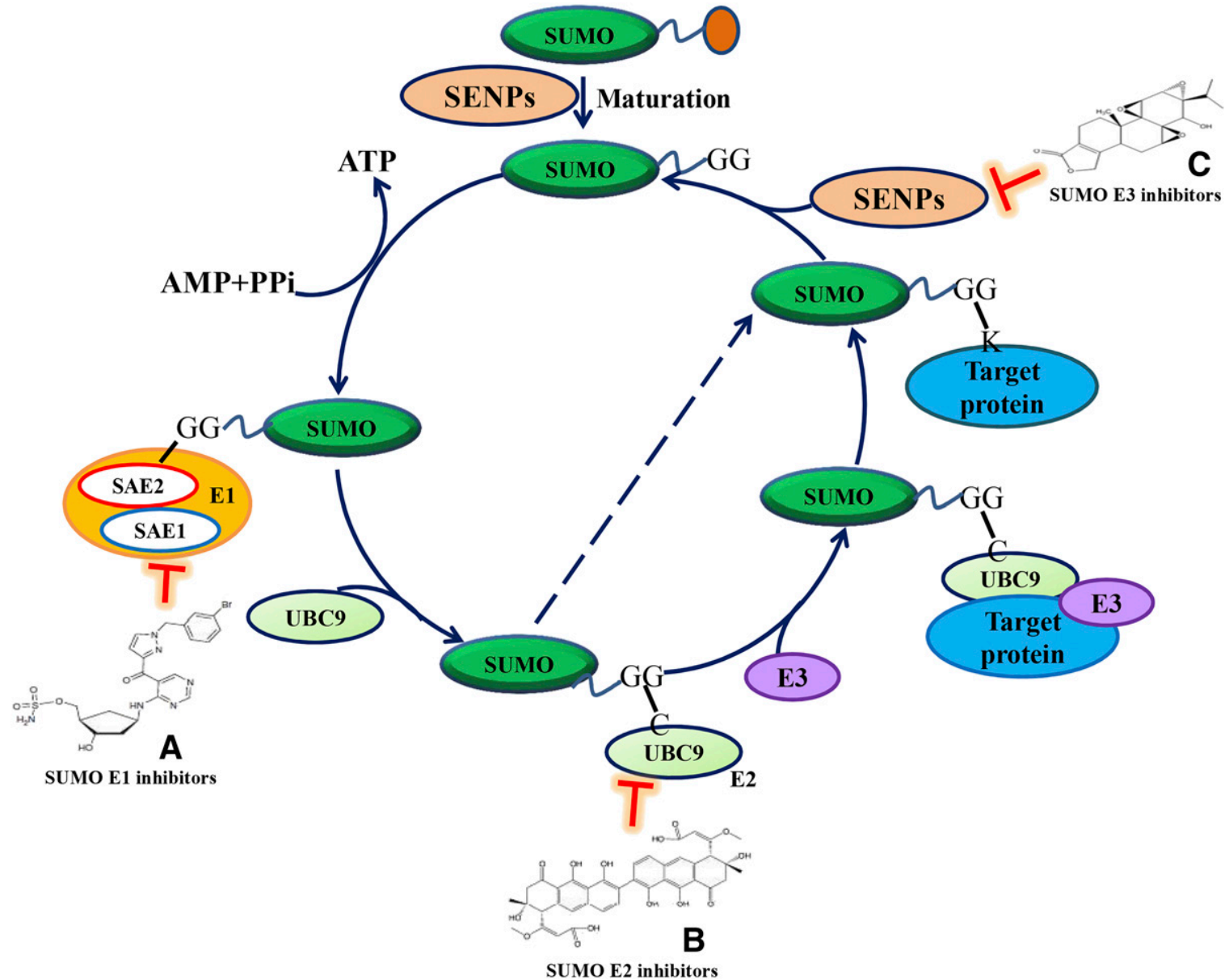

Fig. 1. Small-molecule inhibitors that target the protein SUMOylation process. Protein SUMOylation is a multistep enzymatic cascade reaction. First, all SUMO paralogues are synthesized as preproteins that are cleaved by SENPs to expose a carboxy-terminal diglycine (GG) motif. An ATP-requiring activation step by the heterodimeric SUMO E1 generates a SUMO-SUMO E1 thioester. SUMO is then transferred to the SUMO E2, again forming a thioester. This last step usually requires a SUMO E3 to form an isopeptide bond between the SUMO C terminus and a Lys within the target protein. SUMO E2 promotes SUMOs onto Lys residues of target substrate even in the absence of an SUMO E3. The small-molecule inhibitors targeting SUMOylation-associated enzymes can block the subsequent SUMO modification biochemical process. (A-C) Chemical structure of three inhibitors (ML-792, spectomycin B1, and triptolide).

protein (Yang et al., 2017). Generally, SENPs, SUMO E1, SUMO E2, and SUMO E3 are four key enzymes to in turn perform cleaving, activating, transferring, and ligating of SUMO to Lys residue of a substrate protein.

Protein SUMOylation is a dynamic regulation in physiologic conditions, and the imbalance of SUMOylation and de-SUMOylation associates with the occurrence and progression of cancer and other diseases (Fig. 2) (Liang et al., 2017; Seeler and Dejean, 2017; Yang et al., 2017). To date, due to the importance of SUMOylation in regulating diverse biologic systems in carcinogenesis, several novel small-molecule inhibitors targeting SUMOylation have been developed as promising anticancer compounds. In this review, we summarize the latest research progresses of small-molecule inhibitors by targeting SUMOylation-associated enzymes as highly potent anticancer compounds.

\section{Small-Molecule Inhibitors Targeting Protein SUMOylation-Associated Enzymes}

The expression of SENPs, SUMO E1, SUMO E2, and SUMO E3 is usually increased in numerous cancers (Coppola et al.,
2009; Bellail et al., 2014; Seeler and Dejean, 2017). The SUMO pathway components are involved in various tumors (Coppola et al., 2009; Bellail et al., 2014; Liu et al., 2015; Seeler and Dejean, 2017). The SUMO pathway components are involved in various human cancers (Table 1), so small-molecule inhibitors targeting protein SUMOylation-associated enzymes exhibit promising antitumor activities.

SENP and Its Anticancer Inhibitors. SUMOylation is a reversible and dynamic process between protein SUMOylation and deSUMOylation for normal cellular activities (Yang et al., 2017). SENPs, as cysteine proteases, cleave the precursor or inactive form of SUMO at the $\mathrm{C}$ terminus via its hydrolase activity to expose two GG residues. Alternatively, SENPs can catalyze the deconjugation of SUMO molecules from a target protein. SENPs include six members, including SENP1, SENP2, SENP3, SENP5, SENP6, and SENP7. Among these SENPs, SENP1 is widely increased in various cancers (Table 1) (Zuo and Cheng, 2009; Xu et al., 2011). It plays an important role in cancer occurrence (Bawa-Khalfe et al., 2007), development (Bawa-Khalfe et al., 2010), and metastasis (Wang et al., 2013). For instance, SENP1 enhances vascular endothelial growth factor production by regulating the stability of hypoxia-inducible factor 1 (Xu et al., 2010), which is 


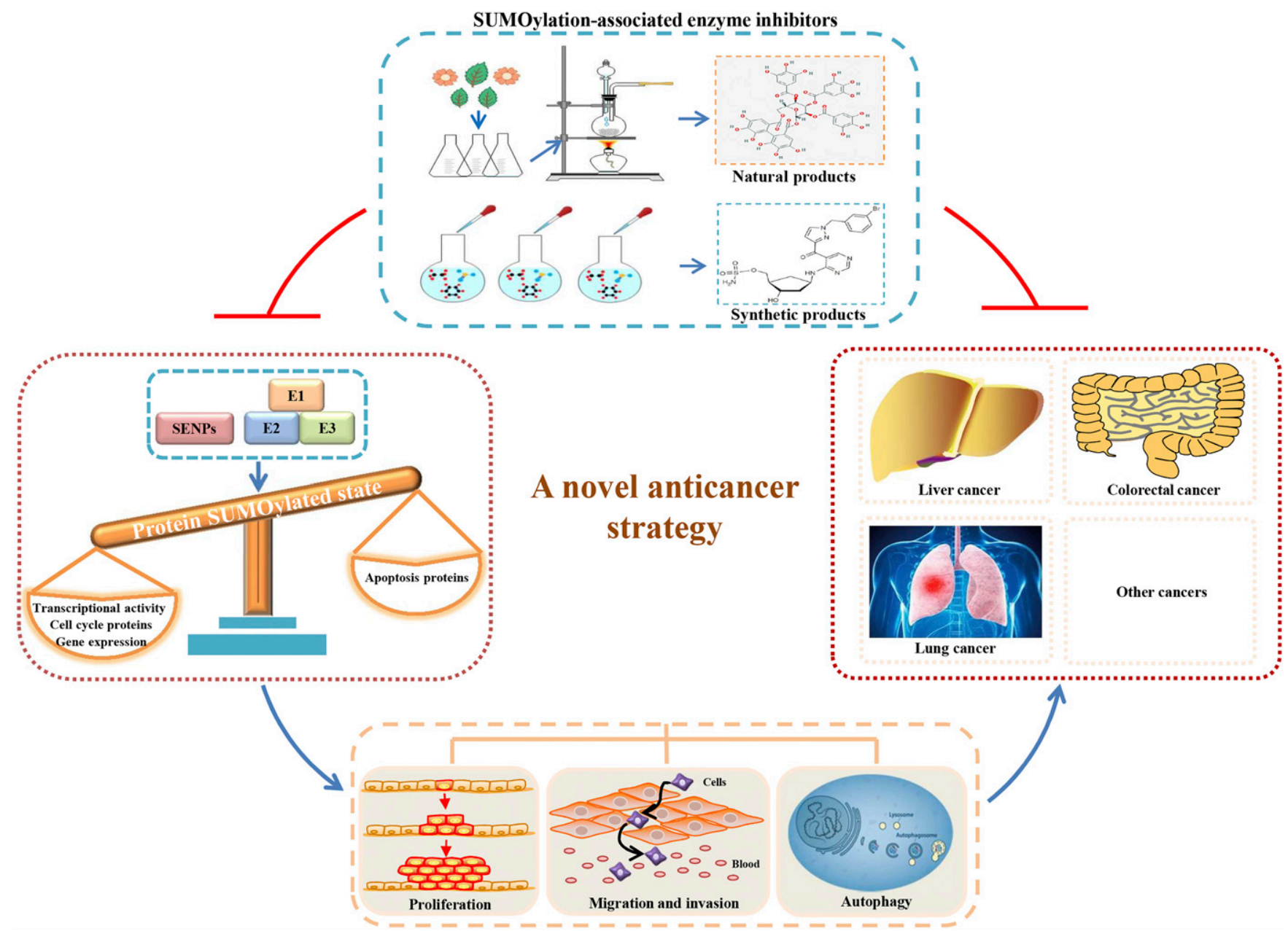

Fig. 2. Novel anticancer drug discovery strategy based on SUMOylation-associated enzyme inhibitors. The high expression of SUMO E1, SUMO E2, SUMO E3, and SENPs in cancer cells breaks the functional balance of SUMOylated and deSUMOylated proteins, which changes gene transcription activity, gene expression, and cell biologic behaviors including cell proliferation, migration, invasion, and autophagy, eventually leading to the occurrence and progression of cancers. The natural or synthetic inhibitors targeting SUMOylation-associated enzymes have good anticancer activities through inhibiting protein SUMOylation.

essential for cancer cell proliferation (Xu et al., 2011). In addition, the up-regulation of SENP1 is positively linked with lymph node metastasis and tumor aggressiveness of pancreatic ductal adenocarcinoma (Ma et al., 2014).

Except for SENP2, which decreases in bladder cancer (Tan et al., 2013) and hepatocellular carcinoma (HCC) (Shen et al., 2012), other SENP members such as SENP3 and SENP5 are also up-regulated in multiple cancers, including neuroblastoma (Xiang-Ming et al., 2016), multiple myeloma (Xu et al., 2015), gastric cancer (Ren et al., 2014), oral squamous cell carcinoma (Sun et al., 2013), and breast cancer (Cashman et al., 2014). Generally, the abnormal expression levels of SENPs can serve as tumor biomarkers.

To date, several SENP1 inhibitors have exhibited good anticancer activities in vitro (Table 2 ). These inhibitors mainly include benzodiazepine-based peptidomimetic covalent compounds (Qiao et al., 2011), SUMO-derived peptide-based covalent inhibitors (Albrow et al., 2011), and the noncovalent 2-(4-chlorophenyl)-2oxoethyl 4-benzamidobenzoates (Chen et al., 2012b) and 1-[4-( $N$ benzylamino) phenyl]-3-phenylureas (Uno et al., 2012).

To improve anticancer potency, several compounds have been designed to obtain micromolar levels of antitumor activities. For example, compound $13 \mathrm{~m}$ has $3.5 \mu \mathrm{M}$ of $\mathrm{IC}_{50}$ through in silico screening and rational drug design (Zhao et al., 2016), and the compound 3 is obtained with $3.3 \mu \mathrm{M}$ of IC50 (Xie et al., 2016). However, these inhibitors need great improvement in order for them to be useful for in-depth in vivo studies.

The SENP1 inhibitors not only are designed through chemical synthesis but also are derived from natural products. Triptolide, extracted from medicinal herbs, down-regulates SENP1 and inhibits prostate cancer cell proliferation (Huang et al., 2012). Similarly, a natural pentacyclic triterpenoid named Momordin Ic also induces cell cycle arrest and cell apoptosis to suppress prostate cancer cell growth by inhibiting of SENP1 (Wu et al., 2016). So SENP1 may serve as an attractive drug target for developing new cancer therapeutics.

In addition to SENP1, SENP2 has been confirmed to be a molecular target for chemical drugs that effectively enhance SUMO conjugation and suppress SENP2 activity. For example, compound 1, 2, 5-oxadiazole, has potential for development into a novel SENP2-targeting therapeutic agent against various diseases (Kumar et al., 2014). In addition, cytoprotective molecules are being developed as novel stroke therapies 


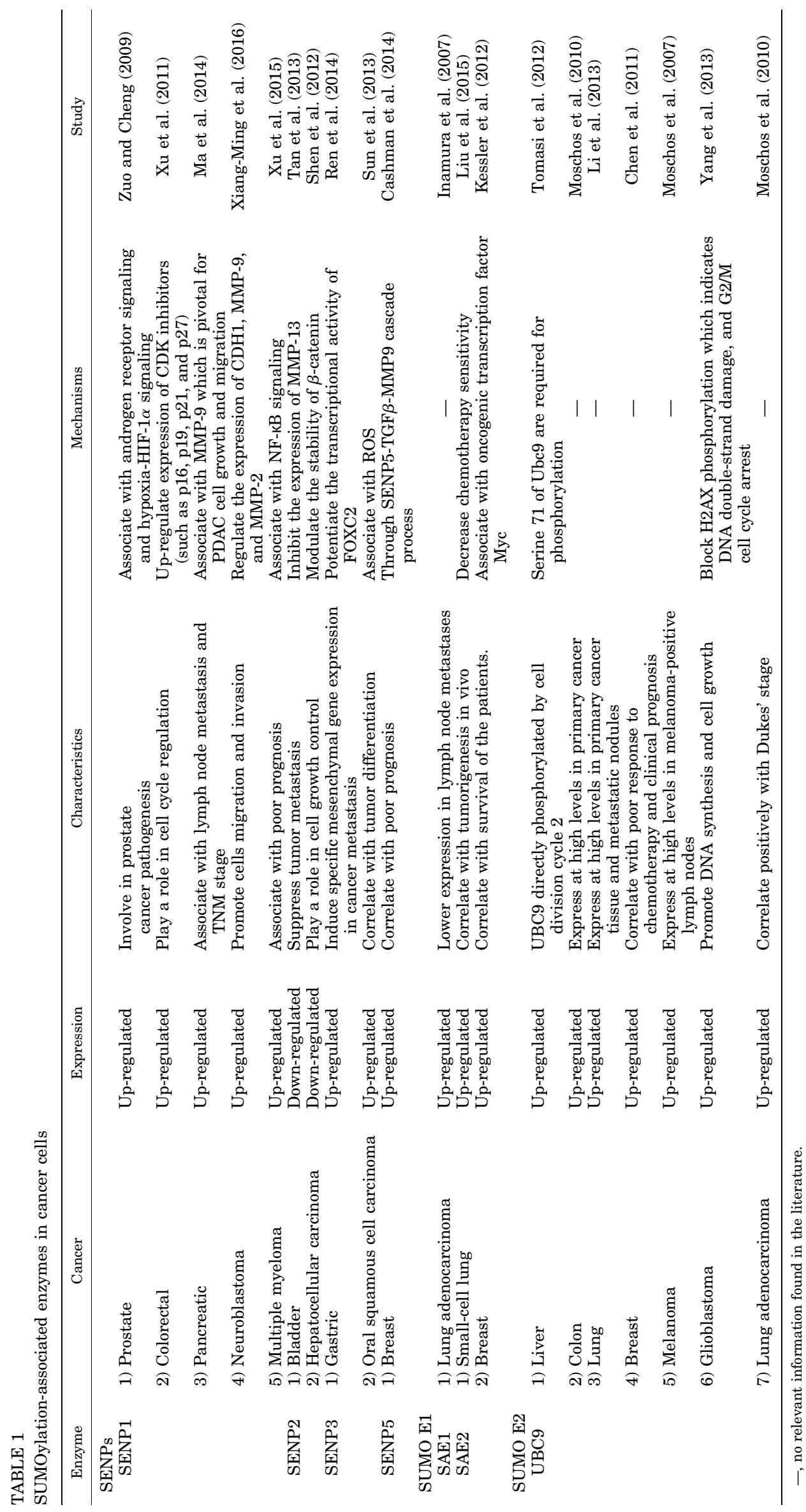




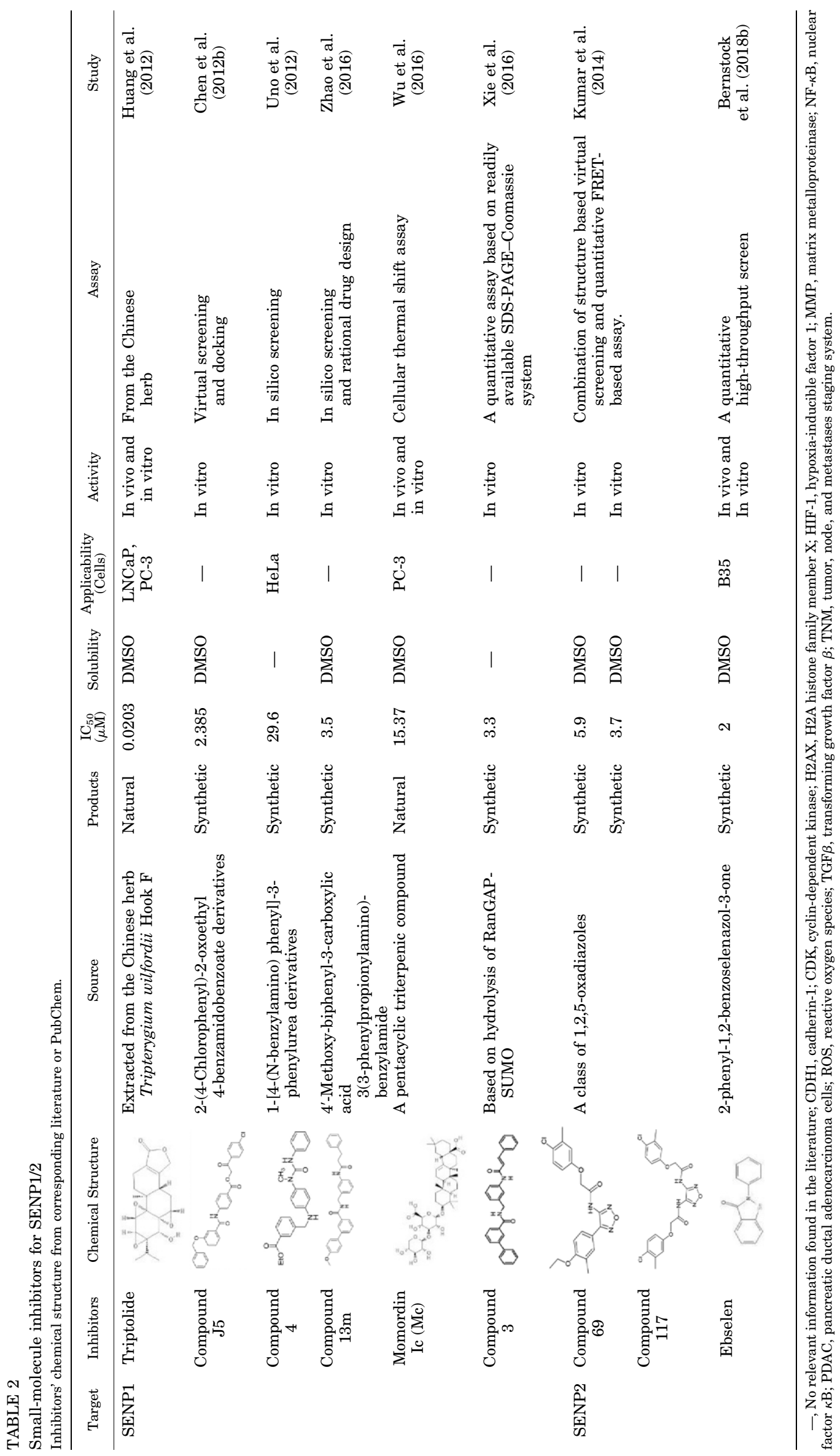




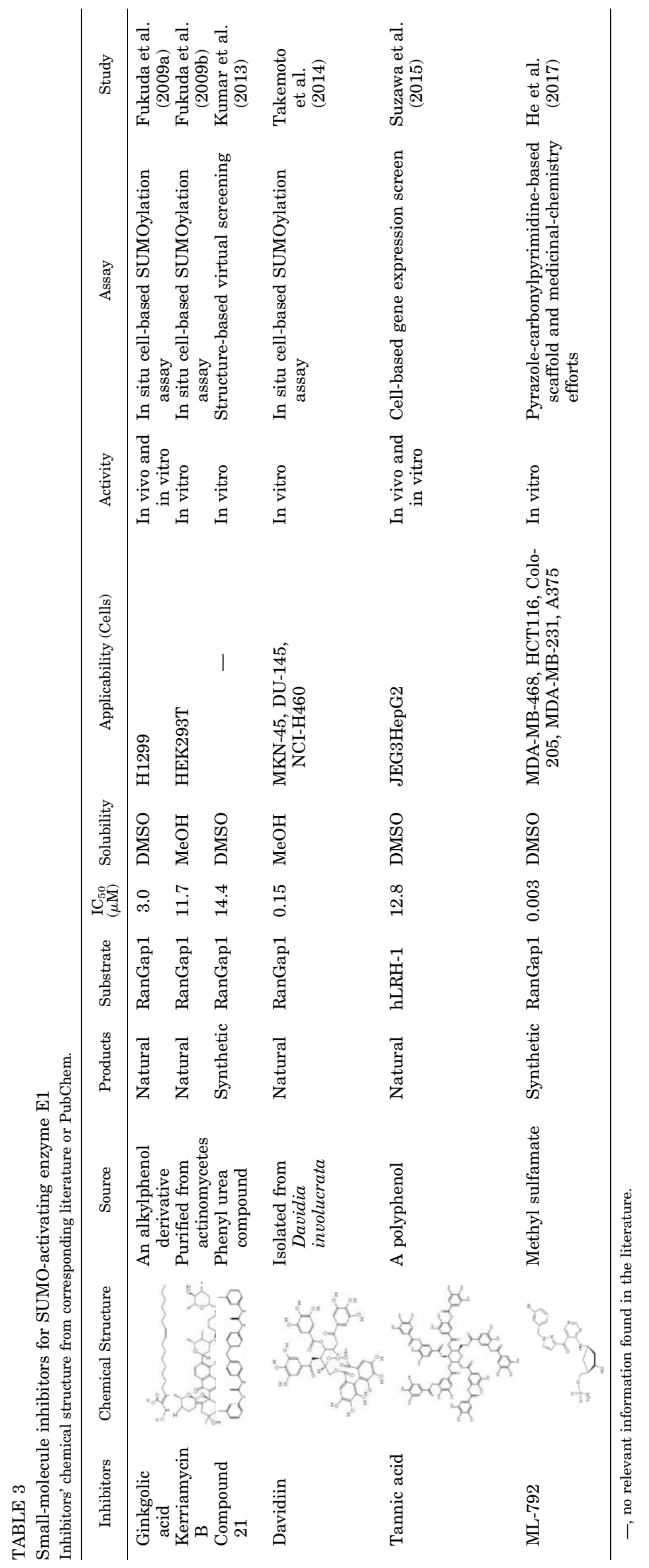


by quantitative high-throughput screening (Bernstock et al., 2018b).

SUMO-E1 and Its Anticancer Inhibitors. SUMO E1, an activating enzyme in SUMOylation pathway, is a heterodimer consisting of SUMO-activating enzyme subunit 1 (SAE1) and subunit 2 (SAE2), which has a particular significance due to its enhancing cell metastasis in tumorigenesis (Table 1). The increased SUMO enzymes are closely associated with pathogenesis of HCC; higher levels of SUMO E1 indicate lower survival rates of HCC patients (Lee and Thorgeirsson, 2004). Knock-down of SAE2 inhibits cancer malignancy and increases chemotherapy sensitivity of small cell lung cancer (Liu et al., 2015). Breast cancer patients with lower expression of SAE1/SAE2 have significantly fewer cases of metastatic cancer and increased survival compared with patients with higher SAE1/SAE2 levels (Kessler et al., 2012). SAE1/SAE2 is also up-regulated in lung adenocarcinoma (Inamura et al., 2007).

There has been growing interest in investigating selective SUMO E1-targeting inhibitors (Table 3). For example, several natural products-including ginkgolic acid (Fukuda et al., 2009a), kerriamycin B (Fukuda et al., 2009b), and davidiin (Takemoto et al., 2014)—are confirmed to inhibit SUMO E1 by blocking formation of SUMO E1-SUMO intermediate. Among these natural products, ginkgolic acid is the most widely used and commercially available chemical regent that targets global SUMOylation, but its inhibitory effects on cells can vary greatly depending on the assay and a measured substrate. Tannic acid has been identified as targeting human liver receptor homolog-1 as a general nontoxic SUMOylation inhibitor via cell-based screening (Suzawa et al., 2015).

Most natural SUMO E1 inhibitors function in the micromolar range except for davidiin, which inhibits at submicromolar concentrations. To address this limitation, other synthetic SAE inhibitors-compound 21 (Kumar et al., 2013) and ML-792 ([(1R,2S,4R)-4-[[5-[1-[(3-bromophenyl)methyl] pyrazole-3-carbonyl]pyrimidin-4-yl]amino]-2-hydroxycyclopentyl]methyl sulfamate) (He et al., 2017) - have been developed to inhibit cancer cell growth with good selective activity. ML-792 forms a covalent adduct with SUMO at its $\mathrm{C}$ terminus, which inhibits the enzyme activity of SAE2 at nanomolar concentrations (He et al., 2017). Therefore, ML-792 selectively blocks SAE activity in treating MYC-amplified tumors.

SUMO-E2 and Its Anticancer Inhibitors. Ubiquitinconjugating enzyme 9 (Ubc9) is a sole SUMO E2 in the SUMOylation pathway, which promotes SUMO transferring into Lys residues of a target substrate even in the absence of SUMO E3 (Gareau and Lima, 2010). Ubc9 is increased in many cancers (Seeler and Dejean, 2017), including advanced melanomas, head and neck tumor, lung tumor, HCC, colon cancer, breast cancer, and glioblastoma (Ahn et al., 2001; Moschos et al., 2007, 2010; Wu et al., 2009; Chen et al., 2011; Tomasi et al., 2012; Li et al., 2013; Yang et al., 2013). Inhibition of Ubc9 expression sensitizes cytotoxic effects of chemotherapeutic drugs against melanoma cells (Ahn et al., 2001; Moschos et al., 2007). Ubc9 stability and its expression are increased by cell division cycle 2 (Cdc2)-mediated phosphorylation in HCC (Tomasi et al., 2012). Therefore, detection of Ubc9 expression level could be helpful to early diagnosis of adenocarcinoma, HCC, and other cancers.

Suppression of Ubc9 by small-molecule inhibitors represents a potential or emerging strategy. We have summarized 
TABLE 5

The pros and cons of SUMO inhibitors

\begin{tabular}{ll}
\hline \multicolumn{2}{c}{ Protein SUMOylation Inhibitors } \\
\hline \multicolumn{2}{c}{ Pros } \\
\hline $\begin{array}{l}\text { Availability, compounds derived from natural products or synthetic } \\
\text { chemicals }\end{array}$ & Unclear molecular mechanism of anticancer \\
$\begin{array}{l}\text { Inhibitors target different SUMOylation-associated enzymes } \\
\text { Reduce the level of protein SUMOylation in different cancer }\end{array}$ & Weak activity and low selectivity \\
$\begin{array}{l}\text { Inhibit cell proliferation and migration of several specific cancer cell } \\
\text { lines }\end{array}$ & No high inhibition rate \\
A few inhibitors have been approved to enter clinical trials & No efficient antitumor activities for multiple cancers \\
\hline
\end{tabular}

several Ubc9 inhibitors that suppress human malignant cells in vitro (Table 4). For instance, GSK145A was identified as an Ubc9 inhibitor using a high-throughput assay (Brandt et al., 2013). 2-D08 (2',3',4'-trihydroxyflavone) has been identified as a potent SUMOylation inhibitor by an electrophoretic mobility shift assay (Kim et al., 2013). In addition, spectomycin B1, an antibiotic against gram-positive bacteria (Staley and Rinehart, 1994) that has been identified as a novel SUMOylation inhibitor with direct binding to Ubc9, reduces estrogen receptor-dependent gene expression and suppresses proliferation of breast-cancer cells (Hirohama et al., 2013). Spectomycin B1 is a potential therapeutic agent against hormone-dependent breast cancers (Hirohama et al., 2013). The development of other Ubc9 chemical inhibitors is ongoing through a small-molecule microarray approach (Zlotkowski et al., 2017).

SUMO-E3 and Its Anticancer Inhibitors. SUMO E3 includes the protein inhibitor of activated STAT (PIAS), Ran binding protein 2 (RanBP2), the polycomb protein (Pc2), and other members (Martin et al., 2007). SUMO conjugation is always enhanced in the presence of SUMO E3, but in the absence of SUMO E3 Ubc9 can also promote SUMOs onto Lys residues of a target substrate. Most of current understanding on SUMO E3 functions mainly comes from studies of the PIAS family (Coppola et al., 2009; Chen et al., 2012a), which regulates protein stability and signaling transduction pathways, and controls inflammation (Rabellino et al., 2017). RanBP2, a nuclear pore complex protein that belongs to the second type of SUMO E3, has also been found to increase in multiple myeloma (Felix et al., 2009) and small cell lung cancer (Horio et al., 2010). Therefore, SUMO E3 ligase could be a potential biomarker for cancer and act as a drug target for cancer therapy. However, no small-molecule inhibitor has been designed to effectively inhibit SUMO-E3 enzymes up to now.

\section{Pros and Cons of SUMOylation-Targeting Small- Molecule Inhibitors}

SUMOylation-associated enzyme inhibitors have been derived from natural products or synthetic chemicals. Besides previous biologic activities, a few natural products have been found to have novel anticancer potentials via acting on the SUMO process. For example, kerriamycin B and spectomycin B1 are typical antibiotics against gram-positive bacteria, and ginkgolic acid and tannic acid have good anti-inflammatory or immunosuppressive activities (Staley and Rinehart, 1994; Antonoff et al., 2009). These reagents have been confirmed to have anticancer activities by inhibition of protein SUMOylation.
Although these compounds have yet to be approved for any clinical trials against tumors, they hold great promise for development into efficient therapeutics, thus extending new clinical applications to old drugs. As they are already used in clinical applications, these drugs would present few novel side effects if their disease treatment spectrum were enlarged.

The SUMOylation pathway is an ideal drug target to overcome oncogenic mechanisms that promote a proapoptotic state, but most SUMOylation inhibitors display weak anticancer activity and uncharacterized selectivity in their current stage (Table 5). Therefore, many more drug-like chemical scaffolds are needed to uncover the therapeutic potential of SUMOylation-associated enzyme inhibitors, along with their structural description.

\section{Future Perspectives}

Protein post-translational modification (PTM) is a step in protein biosynthesis, including phosphorylation, glycosylation, ubiquitination, SUMOylation, and other modifications (Kessler and Edelmann, 2011). Among these PTMs, SUMOylation, a competitor of ubiquitination, has become a research hot spot in recent years (Yang et al., 2017). Mass spectrometry is the most sensitive method for identification and quantification of PTMs (Liang et al., 2012), including protein SUMOylation (McManus et al., 2016; Lamoliatte et al., 2017; Lumpkin et al., 2017). More and more SUMOylated proteins have been shown to be involved in the pathogenesis of multiple diseases, including brain ischemia and cancers (Liang et al., 2017; Yang et al., 2017; Bernstock et al., 2018a). Protein SUMOylation mediates in protein-protein interactions, is responsive to DNA damage, changes protein intracellular localization, or directs changes of protein activities (Dou et al., 2011; Hickey et al., 2012). Thus, the SUMOylation pathway has emerged as a promising therapeutic target for drug discovery because of its crucial role in various cancers.

Compared with conventional high-throughput screening, virtual filtering plays a noteworthy role in the identification and optimization of small-molecule inhibitors targeting the SUMO pathway. Through virtual screening in combination with biologic assay (Fukuda et al., 2009a,b; Kumar et al., 2013; Takemoto et al., 2014; Suzawa et al., 2015; He et al., 2017; Bernstock et al., 2018b). GSK145A, 2-D08, and spectomycin B1 inhibit Ubc9 (Brandt et al., 2013; Hirohama et al., 2013; Kim et al., 2013), and several compounds exhibit potential activity against tumors. However, the discovery phase of SUMOylation-associated enzyme inhibitors as a potential therapeutic remains in its infancy. Many of the available 
inhibitors of SUMOylation-associated enzyme display weak activity and uncharacterized selectivity.

It is worth mentioning that a novel inhibitor, ML-792, specifically inhibits SAE at nanomolar concentrations without suppressing any other ubiquitin-activating enzymes or various other ATP-using enzymes (He et al., 2017). Therefore, the SUMO inhibitors with high selectivity facilitate further exploration of the novel cellular functions of protein SUMOylation, which opens a new avenue for specifically targeting SUMOylation as a potential clinical anticancer therapy.

\section{Authorship Contributions}

Wrote or contributed to the writing of the manuscript: Yang, Xia, Wang, Zhao, Sheng, Ye, He, Zhou, Zhu, Xu, Liang.

\section{References}

Ahn JH, Xu Y, Jang WJ, Matunis MJ, and Hayward GS (2001) Evaluation of interactions of human cytomegalovirus immediate-early IE2 regulatory protein with small ubiquitin-like modifiers and their conjugation enzyme Ubc9. $J$ Virol $\mathbf{7 5}$ : 3859-3872.

Albrow VE, Ponder EL, Fasci D, Békés M, Deu E, Salvesen GS, and Bogyo M (2011) Development of small molecule inhibitors and probes of human SUMO deconjugating proteases. Chem Biol 18:722-732.

Antonoff MB, Chugh R, Borja-Cacho D, Dudeja V, Clawson KA, Skube SJ, Sorenson BS, Saltzman DA, Vickers SM, and Saluja AK (2009) Triptolide therapy for neuroblastoma decreases cell viability in vitro and inhibits tumor growth in vivo. Surgery 146:282-290.

Bawa-Khalfe T, Cheng J, Lin SH, Ittmann MM, and Yeh ET (2010) SENP1 induces prostatic intraepithelial neoplasia through multiple mechanisms. J Biol Chem 285: 25859-25866.

Bawa-Khalfe T, Cheng J, Wang Z, and Yeh ET (2007) Induction of the SUMO-specific protease 1 transcription by the androgen receptor in prostate cancer cells. $J$ Biol Chem 282:37341-37349.

Bellail AC, Olson JJ, and Hao C (2014) SUMO1 modification stabilizes CDK6 protein and drives the cell cycle and glioblastoma progression. Nat Commun 5:4234

Bernstock JD, Yang W, Ye DG, Shen Y, Pluchino S, Lee YJ, Hallenbeck JM, and Paschen W (2018a) SUMOylation in brain ischemia: patterns, targets, and translational implications. J Cereb Blood Flow Metab 38:5-16.

Bernstock JD, Ye D, Smith JA, Lee YJ, Gessler FA, Yasgar A, Kouznetsova J, Jadhav A, Wang Z, Pluchino S, et al. (2018b) Quantitative high-throughput screening identifies cytoprotective molecules that enhance SUMO conjugation via the inhibition of SUMO-specific protease (SENP)2. FASEB $J$ 32:1677-1691.

Bettermann K, Benesch M, Weis S, and Haybaeck J (2012) SUMOylation in carcinogenesis. Cancer Lett 316:113-125.

Brandt M, Szewczuk LM, Zhang H, Hong X, McCormick PM, Lewis TS, Graham TI, Hung ST, Harper-Jones AD, Kerrigan JJ, et al. (2013) Development of a highthroughput screen to detect inhibitors of TRPS1 sumoylation. Assay Drug Dev Technol 11:308-325.

Cashman R, Cohen H, Ben-Hamo R, Zilberberg A, and Efroni S (2014) SENP5 mediates breast cancer invasion via a TGF $\beta$ RI SUMOylation cascade. Oncotarget $\mathbf{5}$ : 1071-1082.

Chen P, Zhao D, Sun Y, Huang L, Zhang S, and Yuan Y (2012a) Protein inhibitor of activated STAT-1 is downregulated in gastric cancer tissue and involved in cell metastasis. Oncol Rep 28:2149-2155.

Chen SF, Gong C, Luo M, Yao HR, Zeng YJ, and Su FX (2011) Ubc9 expression predicts chemoresistance in breast cancer. Chin J Cancer 30:638-644.

Chen Y, Wen D, Huang Z, Huang M, Luo Y, Liu B, Lu H, Wu Y, Peng Y, and Zhang J (2012b) 2-(4-Chlorophenyl)-2-oxoethyl 4-benzamidobenzoate derivatives, a novel class of SENP1 inhibitors: virtual screening, synthesis and biological evaluation. Bioorg Med Chem Lett 22:6867-6870.

Coppola D, Parikh V, Boulware D, and Blanck G (2009) Substantially reduced expression of PIAS1 is associated with colon cancer development. J Cancer Res Clin Oncol 135:1287-1291.

Dou H, Huang C, Van Nguyen T, Lu LS, and Yeh ET (2011) SUMOylation and de-SUMOylation in response to DNA damage. FEBS Lett 585:2891-2896.

Enserink JM (2015) Sumo and the cellular stress response. Cell Div 10:4.

Felix RS, Colleoni GW, Caballero OL, Yamamoto M, Almeida MS, Andrade VC Chauffaille MdeL, Silva WA, Jr, Begnami MD, Soares FA, et al. (2009) SAGE analysis highlights the importance of p53csv, ddx5, mapkapk2 and ranbp2 to multiple myeloma tumorigenesis. Cancer Lett 278:41-48.

Fukuda I, Ito A, Hirai G, Nishimura S, Kawasaki H, Saitoh H, Kimura K, Sodeoka M, and Yoshida M (2009a) Ginkgolic acid inhibits protein SUMOylation by blocking formation of the E1-SUMO intermediate. Chem Biol 16:133-140.

Fukuda I, Ito A, Uramoto M, Saitoh H, Kawasaki H, Osada H, and Yoshida M (2009b) Kerriamycin B inhibits protein SUMOylation. J Antibiot (Tokyo) 62:221-224.

Gareau JR and Lima CD (2010) The SUMO pathway: emerging mechanisms that shape specificity, conjugation and recognition. Nat Rev Mol Cell Biol 11: 861-871.

He X, Riceberg J, Soucy T, Koenig E, Minissale J, Gallery M, Bernard H, Yang X, Liao H, Rabino C, et al. (2017) Probing the roles of SUMOylation in cancer cell biology by using a selective SAE inhibitor. Nat Chem Biol 13:1164-1171.

Hecker CM, Rabiller M, Haglund K, Bayer P, and Dikic I (2006) Specification of SUMO1- and SUMO2-interacting motifs. J Biol Chem 281:16117-16127.
Hickey CM, Wilson NR, and Hochstrasser M (2012) Function and regulation of SUMO proteases. Nat Rev $\mathrm{Mol}$ Cell Biol 13:755-766.

Hirohama M, Kumar A, Fukuda I, Matsuoka S, Igarashi Y, Saitoh H, Takagi M, Shin-ya K, Honda K, Kondoh Y, et al. (2013) Spectomycin B1 as a novel SUMOylation inhibitor that directly binds to SUMO E2. ACS Chem Biol 8: 2635-2642.

Horio Y, Osada H, Shimizu J, Ogawa S, Hida T, and Sekido Y (2010) Relationship of mRNA expressions of RanBP2 and topoisomerase II isoforms to cytotoxicity of amrubicin in human lung cancer cell lines. Cancer Chemother Pharmacol 66 $237-243$

Huang W, He T, Chai C, Yang Y, Zheng Y, Zhou P, Qiao X, Zhang B, Liu Z, Wang J, et al. (2012) Triptolide inhibits the proliferation of prostate cancer cells and downregulates SUMO-specific protease 1 expression. PLoS One 7:e37693.

Inamura K, Shimoji T, Ninomiya H, Hiramatsu M, Okui M, Satoh Y, Okumura S, Nakagawa K, Noda T, Fukayama M, et al. (2007) A metastatic signature in entire lung adenocarcinomas irrespective of morphological heterogeneity. Hum Pathol 38 : $702-709$.

Kessler BM and Edelmann MJ (2011) PTMs in conversation: activity and function of deubiquitinating enzymes regulated via post-translational modifications. Cell Biochem Biophys 60:21-38.

Kessler JD, Kahle KT, Sun T, Meerbrey KL, Schlabach MR, Schmitt EM, Skinner $\mathrm{SO}, \mathrm{Xu} \mathrm{Q}, \mathrm{Li} \mathrm{MZ}$, Hartman ZC, et al. (2012) A SUMOylation-dependent transcriptional subprogram is required for Myc-driven tumorigenesis. Science 335: 348-353.

Kim YS, Nagy K, Keyser S, and Schneekloth JS, Jr (2013) An electrophoretic mobility shift assay identifies a mechanistically unique inhibitor of protein sumoylation. Chem Biol 20:604-613.

Kumar A, Ito A, Hirohama M, Yoshida M, and Zhang KY (2013) Identification of sumoylation activating enzyme 1 inhibitors by structure-based virtual screening. $J$ Chem Inf Model 53:809-820.

Kumar A, Ito A, Takemoto M, Yoshida M, and Zhang KY (2014) Identification of 1,2,5-oxadiazoles as a new class of SENP2 inhibitors using structure based virtual screening. J Chem Inf Model 54:870-880.

Lamoliatte F, McManus FP, Maarifi G, Chelbi-Alix MK, and Thibault P (2017) Uncovering the SUMOylation and ubiquitylation crosstalk in human cells using sequential peptide immunopurification. Nat Commun 8:14109.

Lee JS and Thorgeirsson SS (2004) Genome-scale profiling of gene expression in hepatocellular carcinoma: classification, survival prediction, and identification of therapeutic targets. Gastroenterology 127(5 Suppl 1):S51-S55.

Li H, Niu H, Peng Y, Wang J, and He P (2013) Ubc9 promotes invasion and metastasis of lung cancer cells. Oncol Rep 29:1588-1594.

Liang S, Xu Z, Xu X, Zhao X, Huang C, and Wei Y (2012) Quantitative proteomics for cancer biomarker discovery. Comb Chem High Throughput Screen 15:221-231.

Liang Z, Yang Y, He Y, Yang P, Wang X, He G, Zhang P, Zhu H, Xu N, Zhao X, et al. (2017) SUMOylation of IQGAP1 promotes the development of colorectal cancer. Cancer Lett 411:90-99.

Liu X, Xu Y, Pang Z, Guo F, Qin Q, Yin T, Sang Y, Feng C, Li X, Jiang L, et al. (2015) Knockdown of SUMO-activating enzyme subunit 2 (SAE2) suppresses cancer malignancy and enhances chemotherapy sensitivity in small cell lung cancer. $J$ Hematol Oncol 8:67.

Lumpkin RJ, Gu H, Zhu Y, Leonard M, Ahmad AS, Clauser KR, Meyer JG, Bennett EJ, and Komives EA (2017) Site-specific identification and quantitation of endogenous SUMO modifications under native conditions. Nat Commun 8:1171.

Ma C, Wu B, Huang X, Yuan Z, Nong K, Dong B, Bai Y, Zhu H, Wang W, and Ai K (2014) SUMO-specific protease 1 regulates pancreatic cancer cell proliferation and invasion by targeting MMP-9. Tumour Biol 35:12729-12735.

Martin S, Wilkinson KA, Nishimune A, and Henley JM (2007) Emerging extranuclear roles of protein SUMOylation in neuronal function and dysfunction. Nat Rev Neurosci 8:948-959.

McManus FP, Altamirano CD, and Thibault P (2016) In vitro assay to determine SUMOylation sites on protein substrates. Nat Protoc 11:387-397.

Moschos SJ, Jukic DM, Athanassiou C, Bhargava R, Dacic S, Wang X, Kuan SF, Fayewicz SL, Galambos C, Acquafondata M, et al. (2010) Expression analysis of Ubc9, the single small ubiquitin-like modifier (SUMO) E2 conjugating enzyme, in normal and malignant tissues. Hum Pathol 41:1286-1298.

Moschos SJ, Smith AP, Mandic M, Athanassiou C, Watson-Hurst K, Jukic DM, Edington HD, Kirkwood JM, and Becker D (2007) SAGE and antibody array analysis of melanoma-infiltrated lymph nodes: identification of Ubc9 as an important molecule in advanced-stage melanomas. Oncogene 26:4216-4225.

Qiao Z, Wang W, Wang L, Wen D, Zhao Y, Wang Q, Meng Q, Chen G, Wu Y, and Zhou $H$ (2011) Design, synthesis, and biological evaluation of benzodiazepine-based SUMO-specific protease 1 inhibitors. Bioorg Med Chem Lett 21:6389-6392.

Rabellino A, Andreani C, and Scaglioni PP (2017) The role of PIAS SUMO E3-ligases in cancer. Cancer Res 77:1542-1547.

Ren YH, Liu KJ, Wang M, Yu YN, Yang K, Chen Q, Yu B, Wang W, Li QW, Wang J, et al. (2014) De-SUMOylation of FOXC2 by SENP3 promotes the epithelialmesenchymal transition in gastric cancer cells. Oncotarget 5:7093-7104.

Seeler JS and Dejean A (2003) Nuclear and unclear functions of SUMO. Nat Rev Mol Cell Biol 4:690-699.

Seeler JS and Dejean A (2017) SUMO and the robustness of cancer. Nat Rev Cancer 17:184-197.

Shen HJ, Zhu HY, Yang C, and Ji F (2012) SENP2 regulates hepatocellular carcinoma cell growth by modulating the stability of $\beta$-catenin. Asian Pac J Cancer Prev 13:3583-3587.

Staley AL and Rinehart KL (1994) Spectomycins, new antibacterial compounds produced by Streptomyces spectabilis: isolation, structures, and biosynthesis. J Antibiot (Tokyo) 47:1425-1433.

Sun Z, Hu S, Luo Q, Ye D, Hu D, and Chen F (2013) Overexpression of SENP3 in oral squamous cell carcinoma and its association with differentiation. Oncol Rep 29: 1701-1706 
Suzawa M, Miranda DA, Ramos KA, Ang KK, Faivre EJ, Wilson CG, Caboni L, Arkin MR, Kim YS, Fletterick RJ, et al. (2015) A gene-expression screen identifies a nontoxic sumoylation inhibitor that mimics SUMO-less human LRH-1 in liver. eLife 4: e09003.

Takemoto M, Kawamura Y, Hirohama M, Yamaguchi Y, Handa H, Saitoh H, Nakao Y, Kawada M, Khalid K, Koshino H, et al. (2014) Inhibition of protein SUMOylation by davidiin, an ellagitannin from Davidia involucrata. J Antibiot (Tokyo) 67: 335-338.

Tan MY, Mu XY, Liu B, Wang Y, Bao ED, Qiu JX, and Fan Y (2013) SUMO-specific protease 2 suppresses cell migration and invasion through inhibiting the expression of MMP13 in bladder cancer cells. Cell Physiol Biochem 32:542-548.

Tomasi ML, Tomasi I, Ramani K, Pascale RM, Xu J, Giordano P, Mato JM, and Lu SC (2012) S-adenosyl methionine regulates ubiquitin-conjugating enzyme 9 protein expression and sumoylation in murine liver and human cancers. Hepatology 56:982-993.

Uno M, Koma Y, Ban HS, and Nakamura H (2012) Discovery of 1-[4- $(N$ benzylamino)phenyl]-3-phenylurea derivatives as non-peptidic selective SUMOsentrin specific protease (SENP)1 inhibitors. Bioorg Med Chem Lett 22:5169-5173.

Wang Q, Xia N, Li T, Xu Y, Zou Y, Zuo Y, Fan Q, Bawa-Khalfe T, Yeh ET, and Cheng $\mathrm{J}$ (2013) SUMO-specific protease 1 promotes prostate cancer progression and metastasis. Oncogene 32:2493-2498.

Wu F, Zhu S, Ding Y, Beck WT, and Mo YY (2009) MicroRNA-mediated regulation of Ubc9 expression in cancer cells. Clin Cancer Res 15:1550-1557.

Wu J, Lei H, Zhang J, Chen X, Tang C, Wang W, Xu H, Xiao W, Gu W, and Wu Y (2016) Momordin Ic, a new natural SENP1 inhibitor, inhibits prostate cancer cell proliferation. Oncotarget 7:58995-59005.

Xiang-Ming Y, Zhi-Qiang X, Ting Z, Jian W, Jian P, Li-Qun Y, Ming-Cui F, HongLiang X, Xu C, and Yun Z (2016) SENP1 regulates cell migration and invasion in neuroblastoma. Biotechnol Appl Biochem 63:435-440.

Xie W, Wang Z, Zhang J, Wang L, Zhao Y, and Zhou H (2016) Development and evaluation of a highly reliable assay for SUMO-specific protease inhibitors. Bioorg Med Chem Lett 26:2124-2128.

Xu J, Sun HY, Xiao FJ, Wang H, Yang Y, Wang L, Gao CJ, Guo ZK, Wu CT, and Wang LS (2015) SENP1 inhibition induces apoptosis and growth arrest of multiple myeloma cells through modulation of NF- $\kappa \mathrm{B}$ signaling. Biochem Biophys Res Commun 460:409-415.

Xu Y, Li J, Zuo Y, Deng J, Wang LS, and Chen GQ (2011) SUMO-specific protease 1 regulates the in vitro and in vivo growth of colon cancer cells with the upregulated expression of CDK inhibitors. Cancer Lett 309:78-84.

Xu Y, Zuo Y, Zhang H, Kang X, Yue F, Yi Z, Liu M, Yeh ET, Chen G, and Cheng J (2010) Induction of SENP1 in endothelial cells contributes to hypoxia-driven VEGF expression and angiogenesis. J Biol Chem 285:36682-36688.

Yang W, Wang L, Roehn G, Pearlstein RD, Ali-Osman F, Pan H, Goldbrunner R, Krantz M, Harms C, and Paschen W (2013) Small ubiquitin-like modifier 1-3 conjugation [corrected] is activated in human astrocytic brain tumors and is required for glioblastoma cell survival [published correction appears in Cancer Sci (2013) 104:274]. Cancer Sci 104:70-77.

Yang Y, He Y, Wang X, Liang Z, He G, Zhang P, Zhu H, Xu N, and Liang S (2017) Protein SUMOylation modification and its associations with disease. Open Biol 7: 170167.

Zhao Y, Wang Z, Zhang J, and Zhou H (2016) Identification of SENP1 inhibitors through in silico screening and rational drug design. Eur $J$ Med Chem 122: 178-184.

Zlotkowski K, Hewitt WM, Sinniah RS, Tropea JE, Needle D, Lountos GT, Barchi JJ, Jr, Waugh DS, and Schneekloth JS, Jr (2017) A small-molecule microarray approach for the identification of E2 enzyme inhibitors in ubiquitin-like conjugation pathways. SLAS Discov 22:760-766.

Zuo Y and Cheng JK (2009) Small ubiquitin-like modifier protein-specific protease 1 and prostate cancer. Asian J Androl 11:36-38.

Address correspondence to: Dr. Shufang Liang, State Key Laboratory of Biotherapy and Cancer Center, West China Hospital, Sichuan University, and Collaborative Innovation Center for Biotherapy, No. 17, 3rd Section of People's South Road, Chengdu, 610041, People's Republic of China. E-mail: zizi2006@ scu.edu.cn 